chloroform are death by stoppage of respiration and death by stoppage of the heart ; he lays as much stress on the effect on the heart as on the respiration, and he proceeds to affirm that too strong chloroform vapour may very quickly paralyze the heart. This view is, indeed, similar to the one we have already mentioned as taught in the London schools of medicine. It is also well known that death may occur soon after chloroform has begun to be administered, from the heart being affected. If the operation is begun too soon, fainting from pain may supervene, and a fatal result occur: this has always been strongly insisted upon by Dr. Brunton. Surgeon-Major Lawrie says that in such cases it is not the chloroform that acts on the heart, but simply that there is fatal syncope or fainting.

From the large number of experiments on animals which Dr. Brunton has performed in India, in conjunction with the Hyderabad Commission and a medical delegate of the Indian Government, it appears that the "danger from chloroform is asphyxia or an overdose;" there is none whatever from the heart direct. This statement is a distinct reversal of the view generally held in England. It means that chloroform causes a fatal result by affecting the respiration or by too much being taken into the system and affecting the brain; and that there is no direct paralysis of the heart from the chloroform. A perfectly impartial opinion cannot, however, be formed from the scanty records of the investigation which have been as yet received in England. We must wait for fuller details of the experiments before a final judgment can be passed.

It is well, however, to point out that the prevailing view in England has been founded, not only on experiments on the lower animals, but also on the extended clinical observation of two generations of medical men. Clinical observation is not so accurate or so lucid as that of direct experiment, but it has its value, and one by no means to be despised in a case where it is so extensive, and directed to a subject of such great importance, not only to the medical profession, but to the general public, as the question of the administration of chloroform.

\section{ON THE CAVENDISH EXPERIMENT.}

$\mathrm{N}$ the last number of the Proceedings of the Royal 1 Society (vol. xlvi. p. 253), I have given an account of the improvements that I have made in the apparatus of Cavendish for measuring the constant of gravitation. As the principles and some of the details there set out apply very generally to other experiments where extremely minute forces have to be measured, it is possible that an abstract of this paper may be of sufficient interest to find a place in the columns of NATURE.

In the original experiment of Cavendish (Phil. Trans., I798, p. 469), as is well known, a pair of small masses, $m m$ (Fig. I), carried at the two ends of a very long but light torsion rod, are attracted towards a pair of large masses, $M M$, thus deflecting the arm until the torsion of the suspending wire gives rise to a moment equal to that due to the attraction. The large masses are then placed on the other side of the small ones, as shown by the dotted circles, and the new position of rest of the torsion arm is determined. Half the angle between the two positions of rest is the deflection produced by the attracting masses. The actual force which must be applied to the balls to produce this deflection, can be directly determined in dynamical units when the period of oscillation and the dimensions and masses of the moving parts are known. In the original experiment of Cavendish, the arm is 6 feet long, the little masses are balls of lead 2 inches in diameter, and large ones are lead balls I foot in diameter. Since the attraction of the whole earth on the smaller balls only produces their weight, i.e. the force with which they are attracted downwards, it is evident that the balls, $\mathrm{M} \mathrm{M}$, which are insignificant in comparison with the size of the earth, can only exert an extremely feeble attraction. So small is this that it can only be detected when the beam is entirely inclosed in a case to protect it from draughts; when, further, the whole apparatus is placed in a room into which no one must enter, because the heat of the body would warm the case unevenly, and so set up air currents which would have far more influence than the whole attraction to be measured; and when, finally, the period of oscillation is made very great, as, for instance, five to fifteen minutes. In order to realize how small must be the force that will only just produce an observable displacement of the balls, $m m$, it is sufficient to remember that the force which brings them back to their position of rest is the same as the corresponding force in the case of a pendulum which swings at the same rate. Now a pendulum that would swing backwards and forwards in five minutes would have to be about 20,000 metres long, so that in this case a deflection of one millimetre would be produced by a force equal to $\mathrm{I} / 20,000,000$ of the weight of the bob. In the case of a pendulum swinging backwards and forwards once in fifteen minutes the corresponding force would be nine times as small, or $1 / 180,000,000$ of the weight.

In spite of the very small value of the constant of gravitation, Cavendish was able, by making the apparatus on this enormous scale, to obtain a couple which

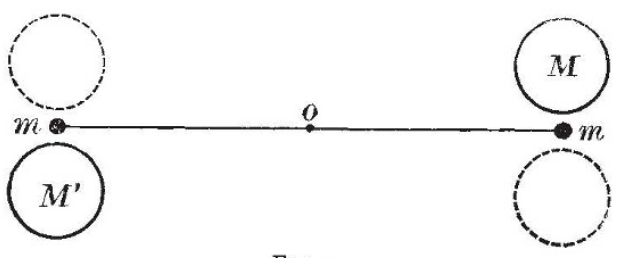

FIG. I.

would produce a definite deflection against the torsion of his suspending wire.

These measures were repeated by Reich (Comptes rendus, 1837, p. 697), and then by Baily (Phil. Mag., r842, vol. xxi. p. I I I), who did not in any important particular improve upon the apparatus of Cavendish, except in the use of a mirror for observing the movements of the beam.

Cornu and Baille (Comptes rendus, vol. 1xxvi. p. 954, vol. lxxxvi. pp. 57 I, 699, IOOI) have modified the apparatus with satisfactory results. In the first place they have reduced the dimensions of all the parts to about onequarter of the original amount. Their beam, an aluminium tube, is only $\frac{1}{2}$ metre long, and it carries at its ends masses of $\frac{1}{4}$ pound each, instead of about 2 pounds, as used by Cavendish. This reduction of the dimensions to about one-quarter of those used previously is considered by them to be one of the advantages of their apparatus, because, as they say, in apparatus geometrically similar, if the period of oscillation is unchanged, the sensibility is independent of the mass of the suspended balls, and is inversely as the linear dimensions. I do not quite follow this, because, as I shall show, if all the dimensions are increased or diminished together, the sensibility will be unchanged. If only the length of the beam is altered and the positions of the large attracting masses, so that they remain opposite to, and the same distance from, the ends of the beam, then the sensibility is inversely as the length. This mistake-for mistake it surely is - is repeated in Jamin's "Cours de Physique," tome iv. ed. iv. p. 18, where, moreover, it is emphasized by being printed in italics.

The other improvements introduced by Cornu and 
Baille are the use of mercury for the attracting masses which can be drawn from one pair of vessels to the other by the observer without his coming near the apparatus, the use of a metal case connected with the earth to prevent electrical disturbances, and the electrical registration of the movements of the index on the scale, which they placed 560 centimetres from the mirror.

The great difficulty that has been met with has been the perpetual shifting of the position of rest, due partly to the imperfect elasticity or fatigue of the torsion wires, but chiefly, as Cavendish proved experimentally, to the enormous effects of air-currents set up by temperature differences in the box, which, with large apparatus, it is impossible to prevent. In every case the power of observing was in excess of the constancy of the effect actually produced. The observations of Cornu are the only ones which are comparable in accuracy with other physical measurements, and these, as far as the few figures given enable one to judge, show a very remarkable agreement between values obtained for the same quantity from time to time.

Soon after I had made quartz fibres, and found their value for producing a very small and constant torsion, I thought that it might be possible to apply them to the Cavendish apparatus with advantage. Prof. Tyndall, in a letter to a neighbour, expressed the conviction that it would be possible to make a much smaller apparatus in which the torsion should be produced by a quartz fibre. The result of an examination of the theory of the instrument shows that very small apparatus ought practically to work, but that in many particulars there is an advantage in departing from the arrangement which has always been employed, conclusions which experiment has fully confirmed.

As I have already stated, the sensibility of the apparatus is, if the period of oscillation is always the same, independent of its linear dimensions. Thus, if there are two instruments in which all the dimensions of one are $n$ times the corresponding dimensions of the other, the moment of inertia of the beam and its appendages will be as $n^{5}: \mathrm{I}$, and, therefore, the torsion also must be as $n^{5}: \mathrm{I}$. The attracting masses, both fixed and movable, will be as $n^{3}: \mathrm{I}$, and their distance apart as $n: \mathrm{I}$. Therefore, the attraction will be as $n^{6} / n^{2}$ or $n^{4}: \mathrm{I}$, and this is acting on an arm $n$ times as long in the large instrument as in the small ; therefore the moment will be as $n^{5}: I$; that is, in the same proportion as the torsion, and so the angle of deflection is unchanged.

If, however, the length of the beam only is changed, and the attracting masses are moved until they are opposite to, and a fixed distance from, the ends of the beam, then the moment of inertia will be altered in the ratio $n^{2}: 1$, while the corresponding moment will only change in the ratio of $n: I$; and thus there is an advantage in reducing the length of the beam until one of two things happens: either it is difficult to find a sufficiently fine torsion thread that will safely carry the beam and produce the required period--and this, I believe, has up to the present time prevented the use of a beam less than $\frac{1}{2}$ metre in length-or else, when the length becomes nearly equal to the diameter of the attracting balls, they then act with such an increasing effect on the opposite suspended balls, so as to tend to deflect the beam in the opposite direction, that the balance of effect begins to fall short of that which would be due to the reduced length if the opposite ball did not interfere. Let this shortening process be continued until the line joining the centres of the masses M M makes an angle of $45^{\circ}$ with the line $m m$; then, without further moving the masses $M \mathrm{M}$, a still greater degree of sensibility can be obtained, provided the period remains unaltered, by reducing the length of the beam $m m$ to half its amount, so that the distance between the centres of $M M$ is $2 \sqrt{2}$ times the new length $m m$, at which point a maximum is reached.
It might be urged against this argument that a difficulty would arise in finding a torsion fibre that would give to a very short beam, loaded with balls that it will safely carry, a period as great as five or ten minutes, and until quartz fibres existed there would have been a difficulty in using a beam much less than a foot long, but it is now possible to hang one only half an inch long and weighing from twenty to thirty grains by a fibre not more than a foot in length, so as to have a period of five minutes. If the moment of inertia of the heaviest beam of a certain length that a fibre will safely carry is so small that the period is too rapid, then the defect can be remedied by reducing the weight, for then a finer fibre can be used, and since the torsion varies approximately as the square of the strength (not exactly, because fine fibres carry heavier weights in proportion), the torsion will be reduced in a higher ratio, and so by making the suspended parts light enough, any slowness that may be required may be provided.

Practically, it is not convenient to use fibres much less than one ten-thousandth of an inch in diameter, and these have a torsion Io, 000 times less than that of ordinary spun glass. A fibre one five-thousandth of an inch in diameter will carry a little over thirty grains.

Since with such small apparatus as I am now using it is easy to provide attracting masses which are very large in proportion to the length of the beam, while with large apparatus comparatively small masses must be made use of owing to the impossibility of dealing with balls of lead of great size, it is clear that much greater deflections can be produced with small than with large apparatus. For instance, to get the same effect in the same time from an instrument with a 6-foot beam that I get from one in which the beam is five-eighths of an inch long, and the attracting balls are 2 inches in diameter, it would be necessary to provide and deal with a pair of balls each 25 feet in diameter, and weighing 730 tons instead of about $\mathbf{I}_{4}^{\frac{3}{4}}$ pound apiece. There is the further advantage in small apparatus that if for any reason the greatest possible effect is desired, attracting balls of gold would not be entirely unattainable, while such small masses as two piles of sovereigns could be used where qualitative effects only were to be shown. Owing to its strongly magnetic qualities, platinum is unsuited for experiments of this kind.

By far the greatest advantage that is met with in small apparatus is the perfect uniformity of temperature which is easily obtained, whereas, with apparatus of large size, this alone makes really accurate work next to impossible. The construction to which this inquiry has led me, and which will be described later, is especially suitable for maintaining a uniform temperature in that part of the instrument in which the beam and mirror are suspended.

With such small beams as I am now using it is much more convenient to replace the long thin box generally employed to protect the beam from disturbance by a vertical tube of circular section, in which the beam with its mirror can revolve freely. This has the further advantage that, if the beam is hung centrally, the attraction of the tube produces no effect, and the troublesome and approximate calculations which have been necessary to find the effect of the box are no longer required. The attracting weights, which must be outside the tube, must be made to take alternately positions on the two sides of the beam, so as to deflect it first in one direction and then in the other. For this purpose they are most conveniently fastened to the inside of a larger metal tube, which can be made to revolve on an axis coincident with the axis of the smaller tube. There are obviously two planes, one containing and one at right angles to the beam, in which the centres of the attracting balls will lie when they produce no deflection. At some intermediate position the deflection will be a maximum. Now, it is a matter of some importance to choose this maximum 
position tor the attracting masses, because, in showing the experiment to an audience, the largest effect should be obtained that the instrument is capable of producing; while in exact measures of the constant of gravitation this position has the further advantage that the only measurement which there is any difficulty in making, viz. the angle between the line joining the large masses and the line joining the small, which may be called the azimuth of the instrument, becomes of little consequence under these circumstances. In the ordinary arrangement the slightest uncertainty in this angle will produce a relatively large uncertainty in the result. I have already stated that if an angle of $45^{\circ}$ is chosen, the distance between the centres of the large balls should be $2 \sqrt{2}$ times the length of the beam, and the converse of course is true. As it would not be possible at this distance to employ attracting balls with a diameter much more than one and a half times the length of the beam, and as balls much larger than this are just as easily made and used, I have found by calculation what are the best positions when the centres of the attracting balls are any distance apart.

If the effect on the nearer ball only is considered, then it is easy to find the best position for any distance of the attracting mass from the axis of motion. Let P (Fig. 2) be the centre of the attracting ball, $\mathrm{N}$ that of the nearer

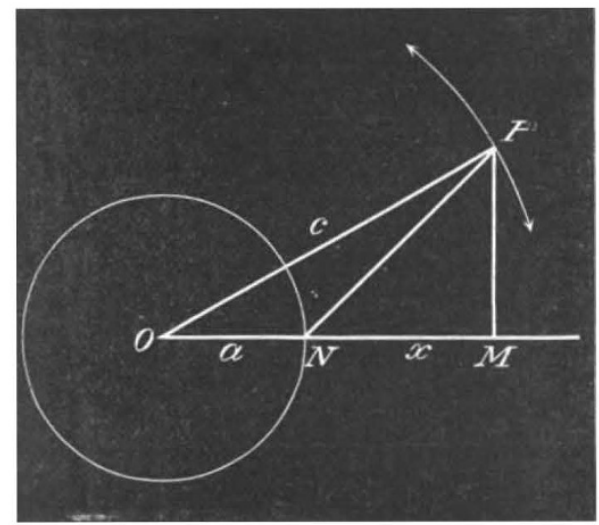

FIG. 2.

attracted ball, $o$ the axis of motion, $c$ and $a$ the distances of $\mathrm{P}$ and $\mathrm{N}$ from $\mathrm{O}$, and $x$ the distance from $\mathrm{N}$ of the foot of the perpendicular from $P$ on oN produced. Then the moment of $\mathrm{N}$ about $\mathrm{O}$ will be greatest when

$$
x^{2}+\frac{3 a^{2}+c^{2}}{a} x=2\left(c^{2}-a^{2}\right),
$$

or what comes to the same thing when

$$
\cos ^{2} \theta+\frac{c^{2}+a^{2}}{c a} \cos \theta=3 \text {. }
$$

Now, as the size of the attracting masses $M$ M is increased, or, as is then necessarily the case, as the distance of their centres from the axis increases, their relative action on the small masses $m m$ at the opposite ends of the beam increases, and so but a small fraction of the advantage is obtained, which the large balls would give if they acted only upon the small balls on their own side. For instance, if the distance between the centres of $\mathrm{M} \mathrm{M}$ is five times the length of the beam, the moment due to the attraction on the opposite small balls is nearly half as great as that on the near balls, so that the actual sensibility is only a little more than half that which would be obtained if the cross action could be prevented.

I have practically overcome this difficulty by arranging the two sides of the apparatus at different levels. Each large mass is at or near the same level as the neighbouring small one, but one pair is removed from the level of the other by about the diameter of the large masses which in the apparatus figured below is nearly five times as great as the distance in plan between the two small masses.

In order to realize more fully the effect of a variety of arrangements, I have, for my own satisfaction, calculated the values of the deflecting forces in an instrument in which the distance between the centres of the attracting balls is five times the length of the beam, for every azimuth and for differences of levels of $0,1,2,3,4$, and 5 times the length of the beam.

The result of the calculation is illustrated by a series of curves in the original paper. The main result, however, is this.

In the particular case which I have chosen for the instrument, $i e$. where the distance between the centres of $M A I$ and the axis, and the difference of level between the two sides are both five times the length of the beam, as seen in plan, and where the diameter of the large masses is 6.4 times the length of the beam, the angle of deflection becomes I 87 times as great as the corresponding angle in the apparatus of Cavendish, provided that the large masses are made of material of the same density in the two cases and the periods of oscillation are the same.

Having now found that with apparatus no bigger than an ordinary galvanometer it should be possible to make an instrument far more sensitive than the large apparatus in use heretofore, it is necessary to show that such a piece of apparatus will practically work, and that it is not liable to be disturbed by the causes which in large apparatus have been found to give so much trouble.

I have made two instruments, of which I shall only describe the second, as that is better than the first, both in design and in its behaviour.

The construction of this is made clear by Fig. 3. To a brass base provided with levelling screws is fixed the vertical brass tube $t$, which forms the chamber in which the small masses $a b$ are suspended by a quartz fibre from a pin at the upper end. These little masses are cylinders ${ }^{1}$ of pure lead I I' 3 millimetres long and 3 millimetres in diameter, and the vertical distance between their centres is 50.8 millimetres. They are held by light brass arms to a very light taper tube of glass, so that their axes are 6.5 millimetres from the axis of motion. The mirror $m$, which is 12.7 millimetres in diameter, plane, and of unusual accuracy, is fastened to the upper end of the glass tube by the smallest quantity of shellac varnish. Both the mirror and the plate-glass window which covers an opening in the tube were examined, and afterwards fixed with the refracting edge of each horizontal, so that the slight but very evident want of parallelism between their faces should not interfere with the definition of the divisions of the scale. The large masses M M are two cylinders ${ }^{1}$ of lead 50.8 millimetres in diameter, and of the same length. They are fastened by screws to the inside of a brass tube, the outline of which is dotted in the figure, which rests on the turned shoulder of the base, so that it may be twisted without shake through any angle. Stops (not shown in the figure) are screwed to the base, so that the actual angle turned through shall be that which produces the maximum deflection. A brass lid made in two halves covers in the outer tube, and serves to maintain a very perfect uniformity of temperature in the inner tube. Neither the masses M M, nor the lid, touch the inner tube. The period of oscillation is 160 seconds.

With this apparatus placed in an ordinary room with

I Cylinders were employed instead of spheres, because they are more easily made and held, and because spheres have no advantage except when absolute calculations have to be made. Also the verticai distance $a b$ was for convenience made only about four times the length $a b$ in plan. 
draughts of air of different temperatures and with a lamp and scale such as are used with a galvanometer, the effect of the attraction can easily be shown to a few, or, with a lime-light, to an audience. To obtain this result with apparatus of the ordinary construction and usual size is next to impossible, on account chiefly of the great disturbing effect of air currents set up by difference of temperature in the case. The extreme portability of the new instrument is a further advantage, as is evident when the enormous weight and size of the attracting masses in the ordinary apparatus are considered.

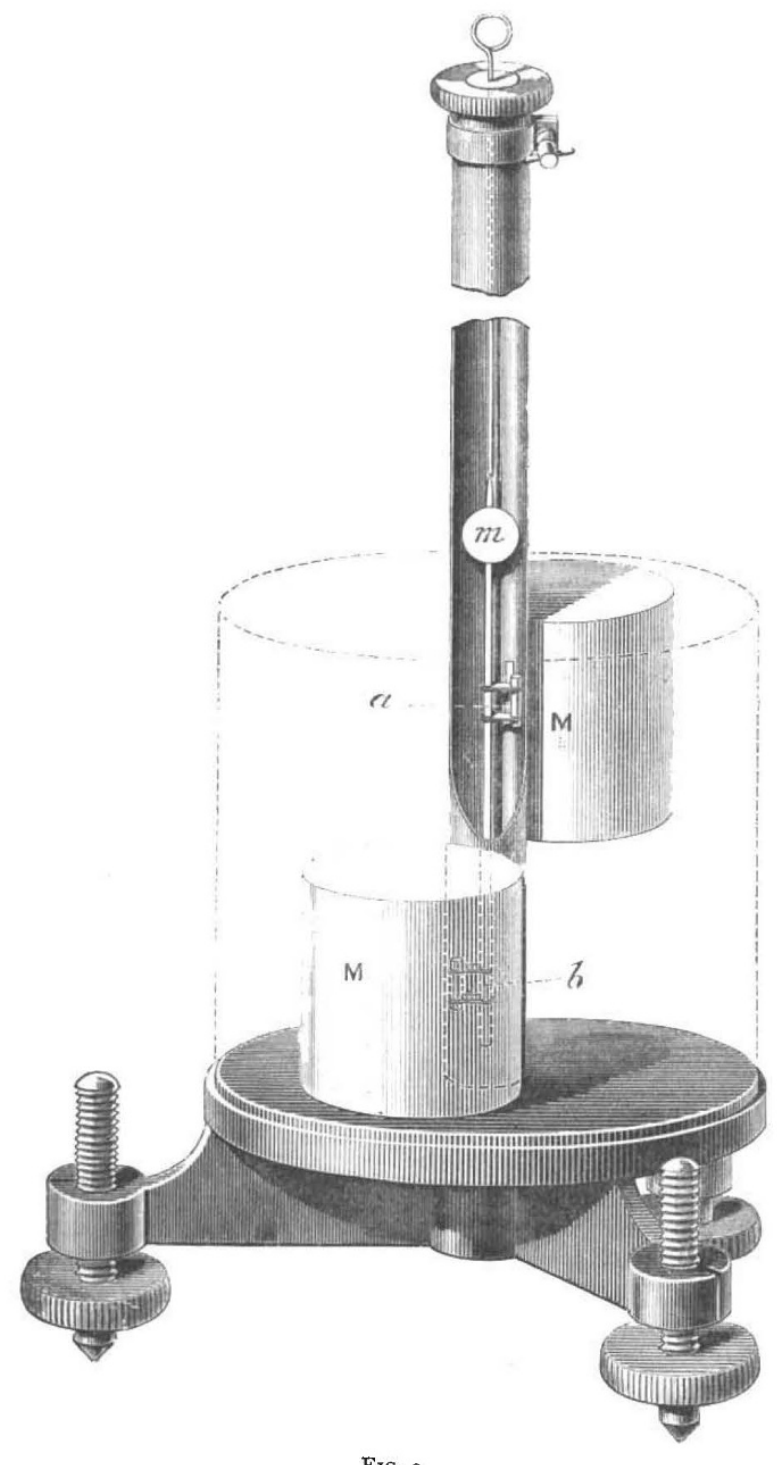

FIG. 3.

However, this result is only one of the objects of the present inquiry. The other object which I had in view was to find whether the small apparatus, besides being more sensitive than that hitherto employed, would also be more free from disturbances and so give more consistent results. With this object I have placed the apparatus in a long narrow vault under the private road between the South Kensington Museum and the Science Schools. This is not a good place for experiments of this kind, for when a cab passes overhead the trembling is so great that loose things visibly move ; however, it is the only place at my disposal that is in any degree suitable. A large drainpipe filled with gravel and cement and covered by a slab of stone forms a fairly good table. The scale is made by etching millimetre divisions on a strip of clear plate glass 80 centimetres long. This is secured at the other end of the vault at a distance of $1053^{\circ} 8$ centimetres from the mirror of the instrument. A telescope $\mathrm{I} 32$ centimetres long with an object-glass 5.08 centimetres in diameter, rests on V's clamped to the wall, with its object-glass 360 centimetres from the mirror. Thus any disturbance that the observer might produce if nearer is avoided, and at the same time the field of view comprises 100 divisions. While the observer is sitting at the telescope he can, by pulling a string, move an albo-carbon light, mounted on a carriage, so as to illuminate any part of the scale that may happen to be in the field of the telescope. The white and steady flame forms a brilliant background on which the divisions appear in black. The accuracy of the mirror is such that the millimetre divisions are clearly defined, and the position of the cross-wire (a quartz fibre) can be read accurately to one-tenth of a division. This corresponds to a movement of the mirror of almost exactly one second of arc.

The mode of observation is as follows: When all is quiet with the large masses in one extreme position, the position of rest is observed and a mark placed on the scale. The masses are moved to one side for a time and then replaced, which sets up an oscillation. The reading of every elongation and the time of every transit of the mark are observed until the amplitude is reduced to 3 or 4 centimetres. The masses are then moved to the other extreme position and the elongations and transits observed again, and this is repeated as often as necessary.

On the evening of Saturday, May I8, six sets of readings were taken, but during the observations there was an almost continuous tramp of art students above, producing a perceptible tremor, besides which two vehicles passed, and coals were twice shovelled in the coal cellar, which is separated from the vault in which the observations were made by only a $4 \frac{1}{2}$-inch brick wall. The result of all this was a nearly perpetual tremor, which produced a rapid oscillation of the scale on the cross-wire, extending over a little more than I millimetre. This increased the difficulty of taking the readings, but to what extent it introduced error I shall not be able to tell until I can make observations in a proper place.

In spite of these disturbances, the agreement between the deflections deduced from the several sets of observations, and between the periods, is far greater than I had hoped to obtain, even under the most favourable conditions. In order to show how well the instrument behaved, I have copied from my note-book the whole series of figures of one set, which sufficiently explain themselves.

\begin{tabular}{|c|c|c|c|c|c|c|c|}
\hline 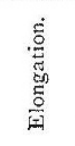 & 苍 & 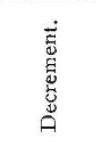 & 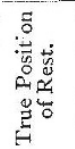 & & & 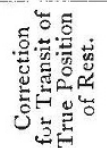 & 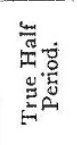 \\
\hline $\begin{array}{l}15 \cdot 05 \\
53 \cdot 20 \\
22 \cdot 48 \\
47 \cdot 28 \\
27 \cdot 28 \\
43 \cdot 40 \\
30 \cdot 42 \\
40 \cdot 88 \\
32 \cdot 50 \\
39 \cdot 27 \\
33 \cdot 80 \\
38 \cdot 25\end{array}$ & $\begin{array}{r}38 \cdot 15 \\
30 \cdot 72 \\
24 \cdot 80 \\
20 \cdot 00 \\
16 \cdot 12 \\
12 \cdot 98 \\
10.46 \\
8 \cdot 38 \\
6 \cdot 77 \\
5 \cdot 47 \\
4 \cdot 45\end{array}$ & $\begin{array}{l}0.805 \\
0.808 \\
0.807 \\
0.807 \\
0.805 \\
0.806 \\
0.802 \\
0.808 \\
0.808 \\
0.814 \\
0.8066\end{array}$ & $\begin{array}{l}36 \cdot 18 \\
36 \cdot 20 \\
36 \cdot 21 \\
36 \cdot 20 \\
36 \cdot 22 \\
36 \cdot 21 \\
36 \cdot 22 \\
36 \cdot 24 \\
36 \cdot 24 \\
36 \cdot 26 \\
36 \cdot 26\end{array}$ & 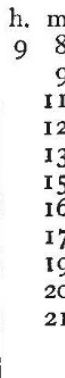 & $\begin{array}{r}\text { s. } \\
255^{\circ} \circ \\
45^{\circ} 5 \\
50^{\circ} \\
25^{\circ} \cdot 8 \\
45^{\circ} \circ \\
60^{\circ} \\
25{ }^{\circ} 0 \\
46.0 \\
40^{\circ} \\
27^{\circ} \circ \\
44^{\circ} 0\end{array}$ & $\begin{array}{l}+0.08 \\
-0.18 \\
+0.24 \\
-0.28 \\
+0.41 \\
-0.47 \\
+0.63 \\
-0.91 \\
+1.13 \\
-1.58 \\
+1.94\end{array}$ & $\begin{array}{l}80 \cdot 2 \\
80 \cdot 2 \\
80 \cdot 0 \\
79 \cdot 9 \\
80 \cdot 1 \\
80 \cdot 1 \\
79 \cdot 5 \\
80 \cdot 5 \\
79 \cdot 8 \\
80 \cdot 5 \\
80 \cdot 08 \\
\end{array}$ \\
\hline
\end{tabular}


It will be noticed that the true position of rest is slightly rising in value, and this rise was found to continue at the rate of 0.36 centimetre an hour during the whole course of the experiment, and to be the same when the large masses were in the positive or negative position. The motion was perfectly uniform, and in no way interfered wlth the accuracy of the experiments. It was due, I believe, to the shellac fastening of the fibre, for I find that immediately after a fibre has been attached, this movement is very noticeable, but after a few days it almost entirely ceases ; it is, moreover, chiefly evident when the fibre is loaded very heavily. At the time that the experiment was made the instrument had only been set up a few hours.

The mean decrement of three positive sets was $0.80 \mathrm{II}$, and of three negative sets, 0.8035 . The observed mean period of three positive sets was $79^{\circ} 98$, and of three negative sets, $80^{\circ} 03$ seconds, from both of which $0^{*} 20$ must be deducted as the time correction for damping.

The deflections, in centimetres, obtained from the six sets of observations taken in groups of three, so as to take into account the effect of the slow change of the position of rest, were as follows:-

$$
\begin{aligned}
& \text { From sets I, 2, and } 3 \ldots \text { I7.66 } \pm 0.0 \mathrm{I} \\
& 2,3 \text {, and } 4 \quad \ldots \quad 17 \cdot 65 \pm 0.02 \\
& 3,4 \text {, and } 5 \text {... } 17.65 \pm 0.02 \\
& 4,5 \text {, and } 60 \text {... } 17.65 \pm 0.02
\end{aligned}
$$

An examination of these figures shows that the deflection is known with an accuracy of about one part in two thousand, while the period is known to the 40ooth part of the whole. As a matter of fact, the discrepancies are not more than may be due to an uncertainty in some of the observations of $\frac{1}{2}$ millimetre or less, a quantity which, under the circumstances, is hardly to be avoided.

The result of these experiments is complete and satisfactory. As a lecture experiment, the attraction between small masses can be easily and certainly shown, even though the resolved force causing motion is, as in the present instance, no more than the $1 / 200,000$ of a dyne (less than $\mathrm{I} / 10,000,000$ of the weight of a grain), and this is possible with the comparatively short half period of 80 seconds. Had it been necessary to make use of such half periods as three to fifteen minutes, which have been employed hitherto, then, even though a considerable deflection were produced, this could hardly be considered a lecture experiment. So perfectly does the instrument behave, that there can be no difficulty in making a fairly accurate measure of the attraction between a pair of No. 5, or, I believe, even of dust shot.

The very remarkable agreement between successive deflections and periods shows that an absolute measure made with apparatus designed for the purpose, but on the lines laid down above, is likely to lead to results of far greater accuracy than any that have been obtained. For instance, in the original experiment of Cavendish there seems to have been an irregularity in the position of rest of one-tenth of the deflection obtained, while the period showed discrepancies of five to fifteen seconds in seven minutes. The experiments of Baily, made in the most elaborate manner, were more consistent, but Cornu was the first to obtain from the Cavendish apparatus results having a precision in any way comparable to that of other physical measurements. The three papers, published by him in the Comptes rendus of 1878 , referred to above, contain a very complete solution of some of the problems to which the investigation has given rise. The agreement between the successive values, decrement, and period is much the same as I have obtained, nevertheless the means of the summer and of the winter observations differ by about I per cent.

I have not referred to the various methods of determining the constant of gravitation in which a balance, whether with the usual horizontal beam, or with a vertical beam on the metronome principle, is employed. They are essentially the same as the Cavendish method, except that there is introduced the friction of the knife-edges and the unknown disturbances due to particles of dust at these points, and to buoyancy, without, in my opinion, any compensating advantage. However, it would appear that if the experiment is to be made with a balance, the considerations which I have advanced in this paper would point to the advantage of making the apparatus small, so that attracting masses of greater proportionate size may be employed, and the disturbance due to convection reduced.

It is my intention, if I can obtain a proper place in which to make the observations, to prepare an apparatus specially suitable for absolute determinations. The scale will have to be increased, so that the dimensions may be determined to a ten-thousandth part at least. Both pairs of masses should, I think, be suspended by fibres or by wires, so that the distance of their centres from the axis may be accurately measured, and so that, in the case of the little masses, the moment of inertia of the beam, mirror, \&c., may be found by alternately measuring the period with and without the masses attached. The unbalanced attractions between the beam, \&c., and the large masses, and between the little masses and anything unsymmetrical about the support of the large masses, will probably be more accurately determined experimentally by observing the deffections when the large and the small masses are in turn removed, than by calculation.

If anything is to be gained by swinging the small masses in a good Sprengel vacuum, the difficulty will not be so great with apparatus made on the scale I have in view, ie. with a beam about 5 centimetres long, as it would with large apparatus. With a view to reduce the considerable decrement, I did try to maintain such a vacuum in the first iustrument, in which a beam $I \cdot 2$ centimetre long was suspended by a fibre so fine as to give a complete period of five minutes, but though the pump would click violently for a day perhaps, leakage always took place before long, and so no satisfactory results were obtained.

With an apparatus such as I have described, but arranged to have a complete period of six minutes, it will be possible to read the scale with an accuracy of $1 / 10,000$ of the deflection, and to determine the time of vibration with an accuracy about twice as great.

I hope early next year, in spite of the difficulty of finding a suitable place to observe in, to prepare apparatus for absolute determinations, and I shall be glad to receive any suggestions which those interested may be good enough to offer.

C. V. Boys.

\section{WILLIAM RAMSAY MCNAB.}

WILLIAM RAMSAY McNAB, M.D., whose sudden death from heart-disease we have already recorded, was born in Edinburgh in November 1844. He was educated at the Edinburgh Academy, and afterwards in the University of that city, obtaining the degree of Doctor of Medicine when twenty-two years of age.

His grandfather and father, in succession, held office as Curators of the Edinburgh Botanic Garden; and the late Dr. McNab early manifested an inherited capacity for botanical work ; for, while still an undergraduate, he was appointed assistant to Prof. Balfour, who then held the Edinburgh botanical chair. He also entered the University of Berlin as a student-in botany under Profs. Braun and Koch, and in pathological anatomy and histology under Prof. Virchow. Three years of his later life were spent in medical practice; but his love of botany was his dominant feeling, and in 1870 he embarked upon a purely biological career, having been then appointed to the Professorship of Natural History 